\title{
Salt supplement increases plasma volume and orthostatic tolerance in patients with unexplained
}

\section{syncope}

\author{
H El-Sayed, R Hainsworth
}

testing though this test is often negative. ${ }^{2}$ Syncope may be provoked by combining tilting with administration of isoprenaline. ${ }^{3}$ This, however, also induces syncope in many asymptomatic volunteers. ${ }^{4}$ We have recently introduced a test that combines head-up tilting with lower body suction. ${ }^{5}$ We believe that this test, which causes progressive reduction in venous return and cardiac output, has a sound physiological basis and we have shown that a stage of the test can be defined which provokes presyncope in only $20 \%$ of asymptomatic volunteers but $85 \%$ of patients with histories strongly suggestive of posturally related syncope ${ }^{6}$ Furthermore, because this test is reproducible, it should be useful in detecting responses in individual patients to therapeutic interventions. Indeed we have already examined the effects of cardiac pacemakers using this test and shown that the time to presyncope was almost identical irrespective of whether or not the heart was being paced.

Treatment of attacks of unexplained syncope is generally unsatisfactory and reports of benefits are often contradictory. For example, treatment with $\beta$-adrenoceptor blocking agents has been claimed by some ${ }^{8-10}$ to relieve symptoms, but others have found no benefit or even an exacerbation of symptoms. ${ }^{11-13}$ The benefits of other agents also seem uncertain. ${ }^{14}$ One form of treatment which has sometimes been recommended to prevent syncope is giving dietary salt supplements. ${ }^{15}$ The rationale for this is that the body's sodium content determines the volume of the extracellular fluid, including that of the plasma. Lately, we found that a subject's orthostatic tolerance is highly significantly correlated with his plasma volume. ${ }^{16}$ It is likely, therefore, that an increase in plasma volume would lead to an increase in orthostatic tolerance. The question remains whether in patients with unexplained syncope an increase in dietary salt intake does increase plasma volume. Reports based on animal studies $^{17}$ or human studies ${ }^{18}$ indicate that salt loading is likely to increase plasma volume. However, there have been no objective studies of the effects of salt in patients with poor orthostatic tolerance and the aim of the present work was to examine, using a double blind protocol, whether salt loading increased both plasma volume and orthostatic tolerance.

Patients and methods

We studied a total of 31 patients complaining of attacks of syncope for which no cause had been detected. These attacks were defined as a
Syncope for no apparent cause in otherwise healthy people is a relatively common clinical problem. ${ }^{1}$ Frequently, patients are subjec to an array of neurological and cardiovascular investigations and, if these are negative and orthostatic hypotension, a diagnosis is made of vasovagal, or neurocardiogenic, syncope. Sometimes the diagnosis can be confirmed by reproducing the hypotension during tilt-table 
transient loss of consciousness and postural tone, with spontaneous prompt and complete recovery. The attacks varied in frequency from only once ever to several attacks per day. The number of attacks ranged from one to 30 (median three) and the period over which they occurred ranged from three to 48 (median 12) months. Clinical examinations in all cases were normal. The patients had been investigated as deemed appropriate by their physicians. All had blood cell counts, electrolyte analyses, resting electrocardiography, 24 or 48 hour Holter monitoring, and cardiac echocardiography. Exercise electrocardiography was undertaken in 25 patients, electroencephalography in eight, computed tomography scan of the head in six, and coronary angiography in five. All results were regarded as normal. No patient was receiving any medical treatment.

This paper is a report of two studies of the effects of salt. The first was a double-blind placebo controlled trial and the second was an open study in which only salt treatment was given. In both studies assessments were made, before and after eight weeks of taking salt or a placebo, of plasma volume, haematocrit, mean corpuscular volume, orthostatic tolerance, baroreceptor sensitivity, and 24 hour urinary sodium excretion. In premenopausal women, the second study was undertaken on the same day of the menstrual cycle as in the initial study and nearest to eight weeks. Between the two assessments, the patients took either salt or placebo. In the double blind study, they took capsules containing $10 \mathrm{mmol}$ sodium chloride or placebo (12 per day). In the open study, they took slow sodium (Ciba) (10 mmol, 12 per day). Patients were informed that the trial was of "a mineral dietary supplement". Salt was not mentioned in the hope that the patients would not modify their own diets.

All patients were instructed to fast, except for water, from midnight before the studies, and to report to the laboratory at $9.00 \mathrm{am}$. The tests were then carried out in the same sequence: plasma volume, baroreceptor sensitivity, orthostatic tolerance.

The studies were approved by the United Leeds Teaching Hospitals Research Ethics Committee and all patients gave informed written consent.

PLASMA VOLUME AND HAEMATOLOGICAL VARIABLES

Plasma volume was estimated by the Evans blue dilution method. ${ }^{19}$ Patients rested supine for 30 minutes, then a venous catheter attached to a plastic tap was inserted into an antecubital vein. After blood was withdrawn for calibration of the spectrophotometer, 15 mg of Evans blue dye (New World Trading Corporation) was injected and thoroughly washed in with isotonic saline. Blood samples were withdrawn $10 \mathrm{~min}$ after injection and every five minutes thereafter to $35 \mathrm{~min}$. The samples were allowed to clot. Then they were centrifuged and the concentration of dye in the serum was determined using a spectrophotometer (Pye Unicam, Cambridge) and compared with known concentrations of dye made in undiluted serum from the same patient. The theoretical concentration of dye in the plasma at the time of injection was calculated by reverse extrapolation of the log concentration decay curve. The total quantity of blood removed from each patient was about $30 \mathrm{ml}$ and this was replaced by an equal volume of saline. The repeatability ( $95 \%$ tolerance limits) of the plasma volume estimates was \pm 62 ml. ${ }^{19}$

The haematocrit was determined by the microhaematocrit method from blood withdrawn immediately before the dye injection. ${ }^{20}$ Blood volume was calculated as: plasma volume/(1 - hematocrit). Mean corpuscular volume was estimated from the initial blood sample using a Coulter counter.

\section{BARORECEPTOR SENSITIVITY}

Subjects reclined supine and were fitted with four standard electrocardiogram leads. After 20 minutes' rest a neck chamber, constructed from thermoplastic, ${ }^{21}$ was fitted round the subject's neck. The subject held his breath in expiration and, after five seconds, a pressure of $0,-20$, or $-40 \mathrm{~mm} \mathrm{Hg}$ was applied for a further five seconds, on two occasions at each pressure. Baroreflex sensitivity was taken as the slope of the maximal prolongation of $R R$ interval of the electrocardiogram against applied chamber pressure. The repeatability of these estimates ( $95 \%$ tolerance limits) was $\pm 0.5 \mathrm{~ms} / \mathrm{mm} \mathrm{Hg}$ ( $\pm 8.5 \%$ of mean values).

\section{ORTHOSTATIC TOLERANCE}

We used the method recently developed and evaluated in our laboratory. ${ }^{5}$ Subjects rested supine for 20 minutes on a specially constructed tilt table with built-on lower body chamber. The subject was then tilted head-up by $60^{\circ}$ for 20 minutes after which, while they

Baseline data (mean (SE)) and responses to salt or placebo

\begin{tabular}{|c|c|c|c|c|}
\hline \multirow[b]{2}{*}{ Variable } & \multicolumn{2}{|l|}{ Salt $(n=10)$} & \multicolumn{2}{|l|}{ Placebo $(n=10)$} \\
\hline & Baseline & Change & Baseline & Change \\
\hline $\begin{array}{l}\text { Weight (kg) } \\
\text { Plasma volume (ml) } \\
\text { Blood volume (ml) } \\
\text { Haematocrit } \\
\text { Mean corpuscular volume (f) } \\
\text { Time to syncope (min) } \\
\text { Baroreceptor sensitivity }(\mathrm{ms} / \mathrm{mm} \mathrm{Hg}) \\
\text { Urinary } \mathrm{Na} \text { (mmol/day) }\end{array}$ & $\begin{array}{c}77 \cdot 1(5 \cdot 5) \\
2930(193) \\
4912(370) \\
0 \cdot 40(0 \cdot 01) \\
90 \cdot 3(0 \cdot 8) \\
20 \cdot 6(1 \cdot 6) \\
5 \cdot 9(0 \cdot 8) \\
128(13)\end{array}$ & $\begin{array}{l}+1 \cdot 7(0.3)^{\star} \\
+185(43)^{\star \star} \dagger \\
+291(56)^{\star \star} \ddagger \\
-0.01(0.005) \\
+0.6(0.3) \\
+8 \cdot 7(1.4)^{\star \star} \dagger \\
-2 \cdot 0(0.4)^{\star \star \dagger} \\
+95(14)^{\star \star} \ddagger\end{array}$ & $\begin{array}{c}67 \cdot 4(2 \cdot 5) \\
2727(163) \\
4391(302) \\
0 \cdot 39(0 \cdot 01) \\
88 \cdot 3(1 \cdot 2) \\
18 \cdot 0(2 \cdot 2) \\
6 \cdot 0(1 \cdot 4) \\
129(18)\end{array}$ & $\begin{array}{l}+0.7(0.4) \\
+97(83) \\
+110(108) \\
+0.004(0.005) \\
+0.2(0.5) \\
+4 \cdot 0(2 \cdot 4) \\
+0.6(0.4) \\
+21(18)\end{array}$ \\
\hline
\end{tabular}

Values after treatment which were significantly different from those before treatment (Wilcoxon signed-rank test): ${ }^{\star} P<0 \cdot 02,{ }^{\star} \mathrm{P}$ $<0.002$. Changes after salt treatment which were significantly different from corresponding changes after placebo (Mann$<0.002$. Changes after salt treatment which
Whitney U test): $\dagger P<0.01, \ddagger P<0.005$. 

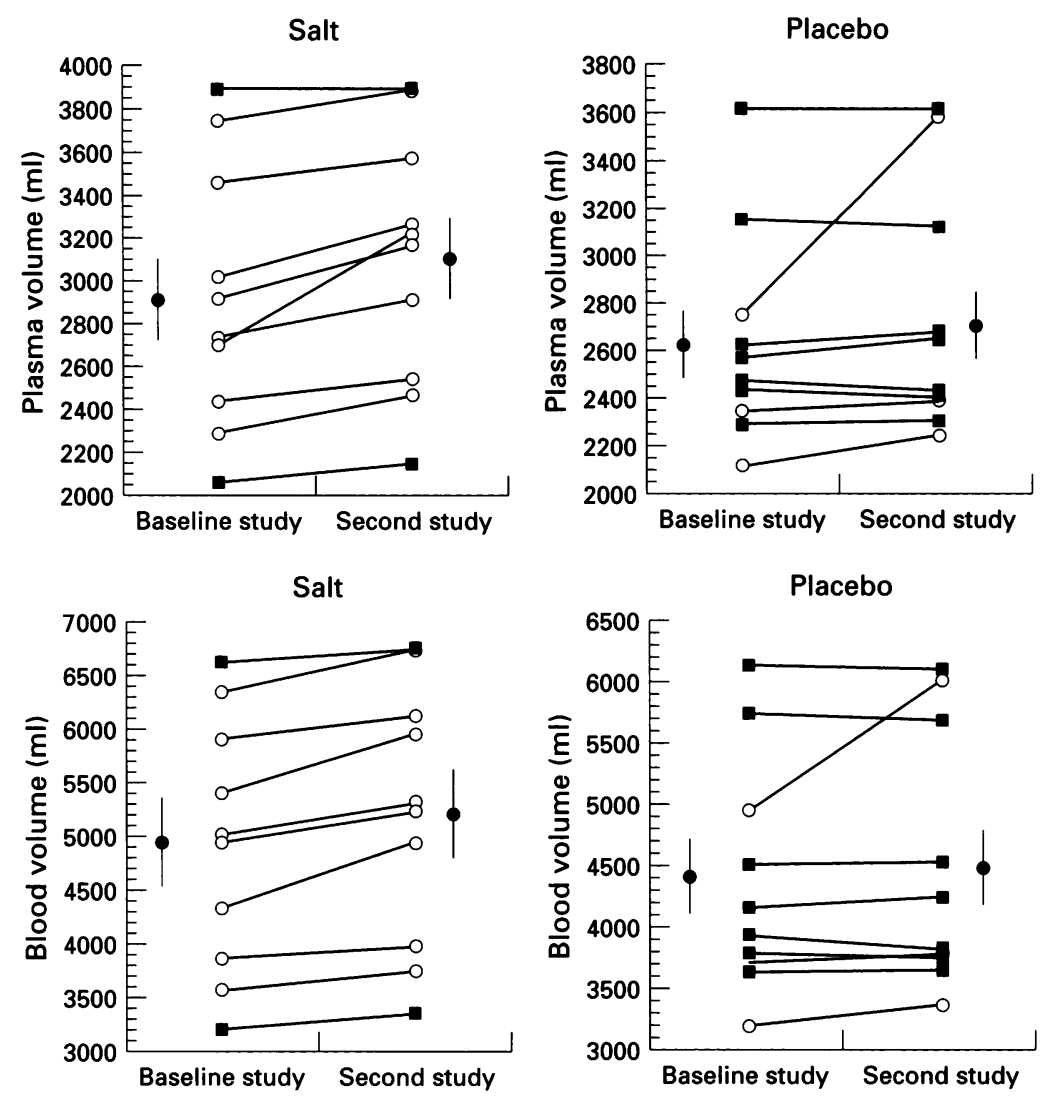

Figure 1 Plasma volume (top) and blood volume (bottom) in patients before and after treatment with salt or placebo. Open circles denote patients in whom the change in plasma volume after treatment was greater than the $95 \%$ tolerance limits of the estimates.
Salt

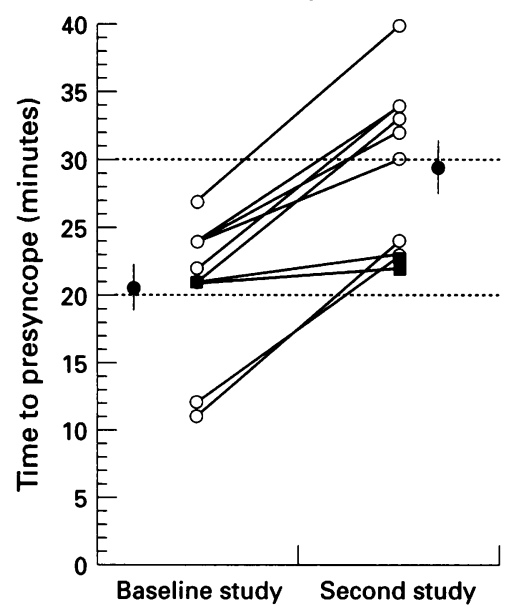

Salt

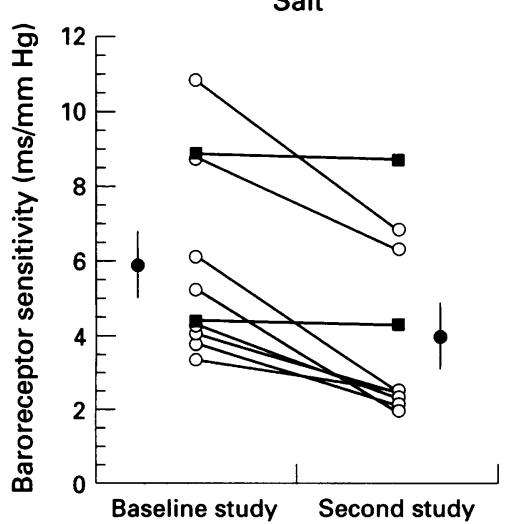

Placebo

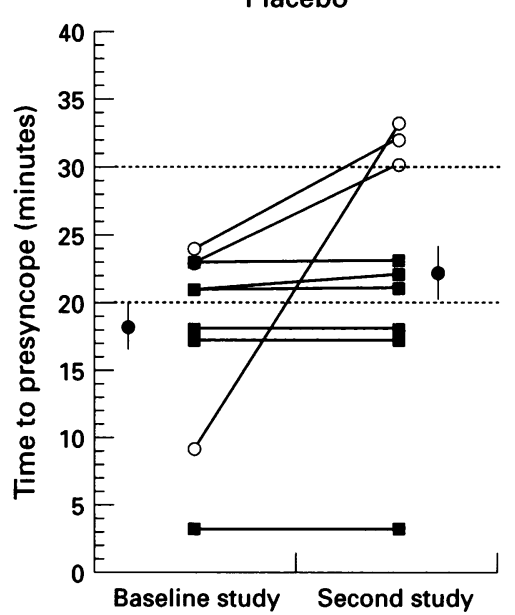

Placebo

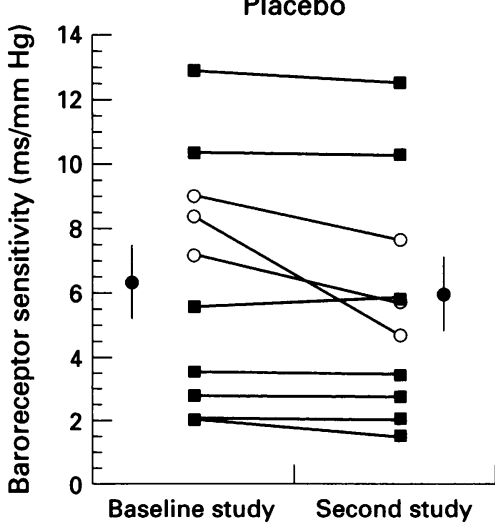

Figure 2 Values of orthostatic tolerance (time to presyncope) and baroreceptor sensitivity in patients before and after salt or placebo treatment. Open and closed circles identify the patients in whom there was and was not a significant increase in plasma volume as shown in fig 1. Note that in top plots, at the time to syncope of $20 \mathrm{~min}$, lower body negative pressure was applied at $20 \mathrm{~mm} \mathrm{Hg}$ and at $30 \mathrm{~min}$ it was increased to $40 \mathrm{~mm} \mathrm{Hg}$. This figure emphasises that all patients in whom plasma volume increased (both salt and placebo groups) also showed increases in orthostatic tolerance and decreases in baroreceptor sensitivity. were still tilted, lower body suction was applied to below the iliac crests at -20 and then $-40 \mathrm{~mm} \mathrm{Hg}$ for 10 minutes at each pressure. Blood pressure was recorded throughout the test from the brachial artery by an automatic sphygmomanometer (Infrasonde, Puritan-Bennett, California) and in a finger maintained at heart level by a Finapres monitor (Ohmeda, Wisconsin). Heart rate was determined from the electrocardiogram. The end point of the test and the measure of orthostatic tolerance was the onset of imminent syncope, seen as a fall in systolic blood pressure to 80 $\mathrm{mm} \mathrm{Hg}$ or less. The repeatability of this test ( $95 \%$ tolerance limits) is $\pm 2.5 \mathrm{~min}$.

\section{Results}

DOUBLE BLIND STUDY

Twenty patients who had been shown to have poor orthostatic tolerance ${ }^{6}$ were randomly allocated to the salt and placebo groups (10 to each). The ages and heights of the patients in the salt and placebo groups (means (SD)) were $41 \cdot 2(4 \cdot 1)$ and $46 \cdot 1(5 \cdot 8)$ years and $171 \cdot 4(3 \cdot 1)$ and $169 \cdot 8(2 \cdot 8) \mathrm{cm}$. Other data are listed in the table. There were no significant differences between the groups in any of the baseline data (Mann-Whitney U test, P > 0.05).

At the second assessment the group taking salt showed significant increases in body weight, plasma and blood volumes, orthostatic tolerance (assessed as the time to onset of syncope), and urinary sodium excretion ( $P<$ 0.05 , Wilcoxon signed-rank test). They also showed a significant decrease in baroreceptor sensitivity. None of these variables changed significantly in the placebo group and all variables in the salt group that changed significantly from the baseline data showed changes that were also significantly different from the corresponding changes in the placebo group (Mann-Whitney test, $P<0.05$ ). The responses are compared in the table.

Responses in individual patients were regarded as significant if they changed beyond the $95 \%$ tolerance limits of the repeatability of measurements. On this basis eight of the 10 patients taking salt but only three of the 10 taking placebo showed significant increases in plasma and blood volumes; the other patients showed no significant responses (fig 1). All the patients from both groups who showed increases in plasma and blood volumes also showed improved tolerance to the orthostatic stress test and decreases in baroreceptor sensitivity (fig 2). The patients in whom the plasma and blood volumes did not change did not show changes in either orthostatic tolerance or baroreceptor sensitivity. The only difference seen between patients who did not respond to salt and those who did was that their baseline salt excretion was higher. The mean 24 hour urinary salt excretion in the responding patients was 105 (range 66-152) $\mathrm{mmol}$ and in those who failed to respond it was 219 and $226 \mathrm{mmol}$. The three patients in the placebo group who had increases in plasma volume and orthostatic tolerance and decreases in baroreceptor sensitivity all showed larger 


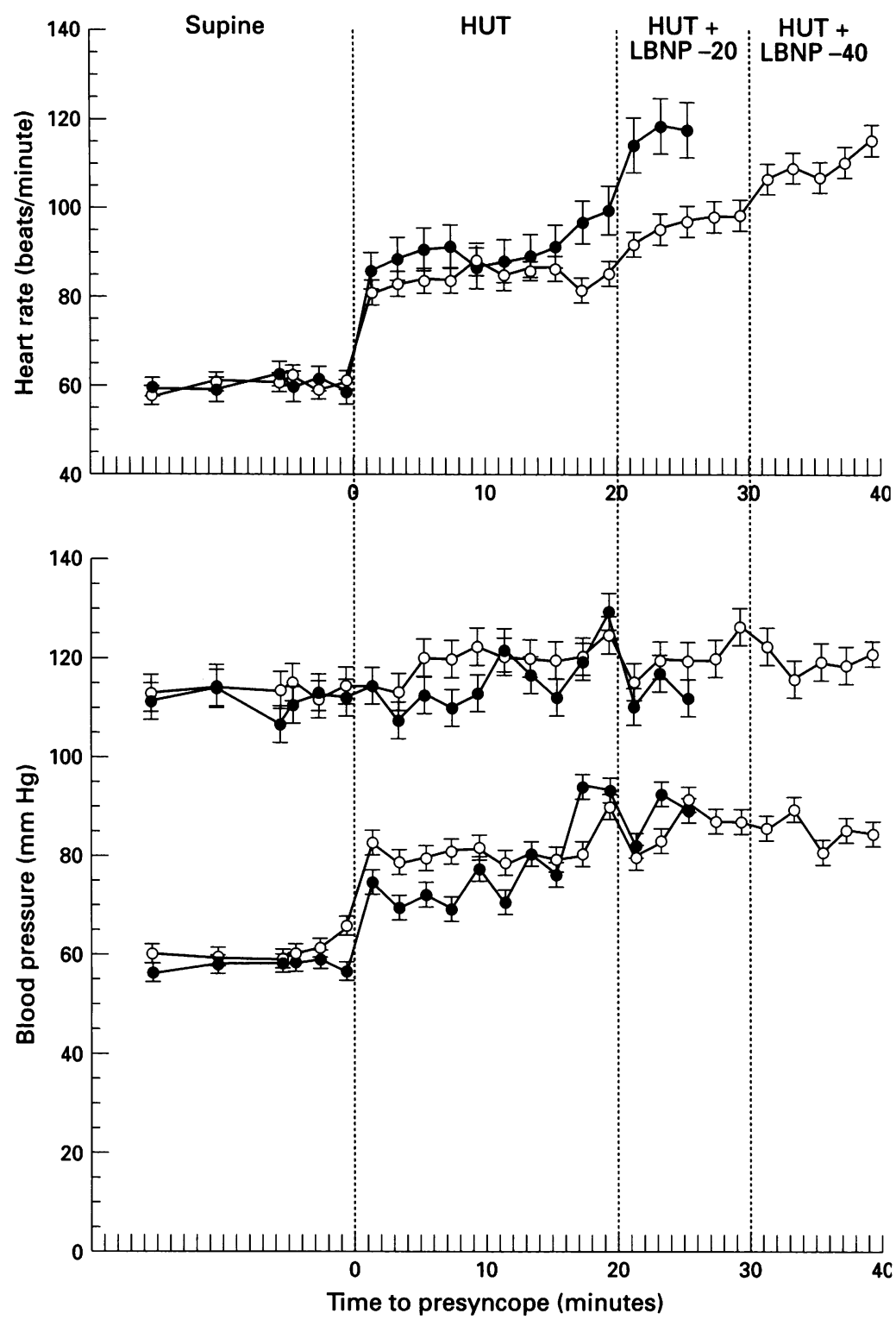

Figure 3 Time course of changes in heart rate and systolic and diastolic blood pressures during the orthostatic stress test in the 21 patients who received salt. Results (means $(S E M)$ ) are of tests before (O) and after (O) treatment. HUT, head up tilt, LBNP, lower body negative pressure. increases in urinary sodium excretion at the second studies than were seen in the placebo patients who did not show changes $(+115$, +83 , and +39 compared with a range of $-36-+7 \mathrm{mmol} / 24 \mathrm{~h}$ ).

\section{OPEN STUDY}

Eleven patients were openly given sodium chloride tablets (slow sodium (Ciba) 120 $\mathrm{mmol} /$ day) and were studied before and after eight weeks of treatment as in the double blind study. There was no significant difference in any of the baseline values in comparison with either group of the double blind study.

Of the 11 patients openly receiving salt, seven showed increases in plasma and blood volumes. These patients also showed increased orthostatic tolerance and decreased baroreceptor sensitivity. As in the double blind study, the only difference in the patients who did not respond to salt was that their baseline sodium excretion was high $(192-262 \mathrm{mmol} /$ $24 \mathrm{~h}$ compared with $45-166 \mathrm{mmol} / 24$ hour in patients who did respond).
RESPONSES OF BLOOD PRESSURE AND HEART RATE

Salt administration had no significant effect on the supine values of blood pressure or heart rate in either of the two groups of patients who were given salt. The mean values and SD in the 21 patients given salt, before and after 8 weeks of treatment were systolic pressure $110.6(10.6)$ and $113.1(9.5) \mathrm{mm} \mathrm{Hg}$, diastolic pressure $60.8(9.7)$ and $61.5(7.5) \mathrm{mm}$ $\mathrm{Hg}$, heart rate $63.4(11.8)$ and $63.7(9.6)$ beats $/ \mathrm{min}$. Values of blood pressures in the head-up tilt position were significantly $(\mathbf{P}<$ 0.01 ) higher after salt administration but were unchanged by placebo. Figure 3 shows the values of heart rate and blood pressure throughout the test, before and after salt administration.

\section{RELATIONS BETWEEN VARIABLES}

Figure 4 shows the relations between the changes in orthostatic tolerance and the change in baroreceptor sensitivity, with the change in plasma volume. This figure, which presents data from all groups of patients including the placebo group shows that all patients in whom plasma volume increased by over $90 \mathrm{ml}$ had significant increases in orthostatic tolerance and significant decreases in baroreceptor sensitivity. Only one of the 13 patients in whom plasma volume changed by less than $90 \mathrm{ml}$ showed an increase in orthostatic tolerance and a decrease in baroreceptor sensitivity.

Figure 5 shows the influence of the baseline salt excretion on the responses of plasma volume and orthostatic tolerance in the 21 patients given salt. The patients in whom the baseline excretion was less than $170 \mathrm{mmol} / 24 \mathrm{~h}$ all showed increases in plasma volume and orthostatic tolerance. Those excreting more than $190 \mathrm{mmol} /$ day did not show significant increases.

\section{SYMPTOMATIC EFFECTS}

Most of the patients who took the salt capsules in the double blind study initially experienced some nausea. In all but one this persisted for less than two weeks, and in no subject did it necessitate withdrawal from the trial. No patient experienced any problems in taking the slow release sodium chloride tablets that were used in the open study. All patients, from both studies in whom increases in plasma volume and orthostatic tolerance were observed, including the three patients taking placebo who also showed changes, claimed to be entirely free from symptoms at the time of the second study. On the other hand, all the patients in whom the test results were unchanged claimed to be unchanged symptomatically.

\section{Discussion}

The principal finding from this study was that administration of salt supplements to patients suffering from attacks of unexplained syncope significantly increased plasma and blood volumes, improved orthostatic tolerance, 

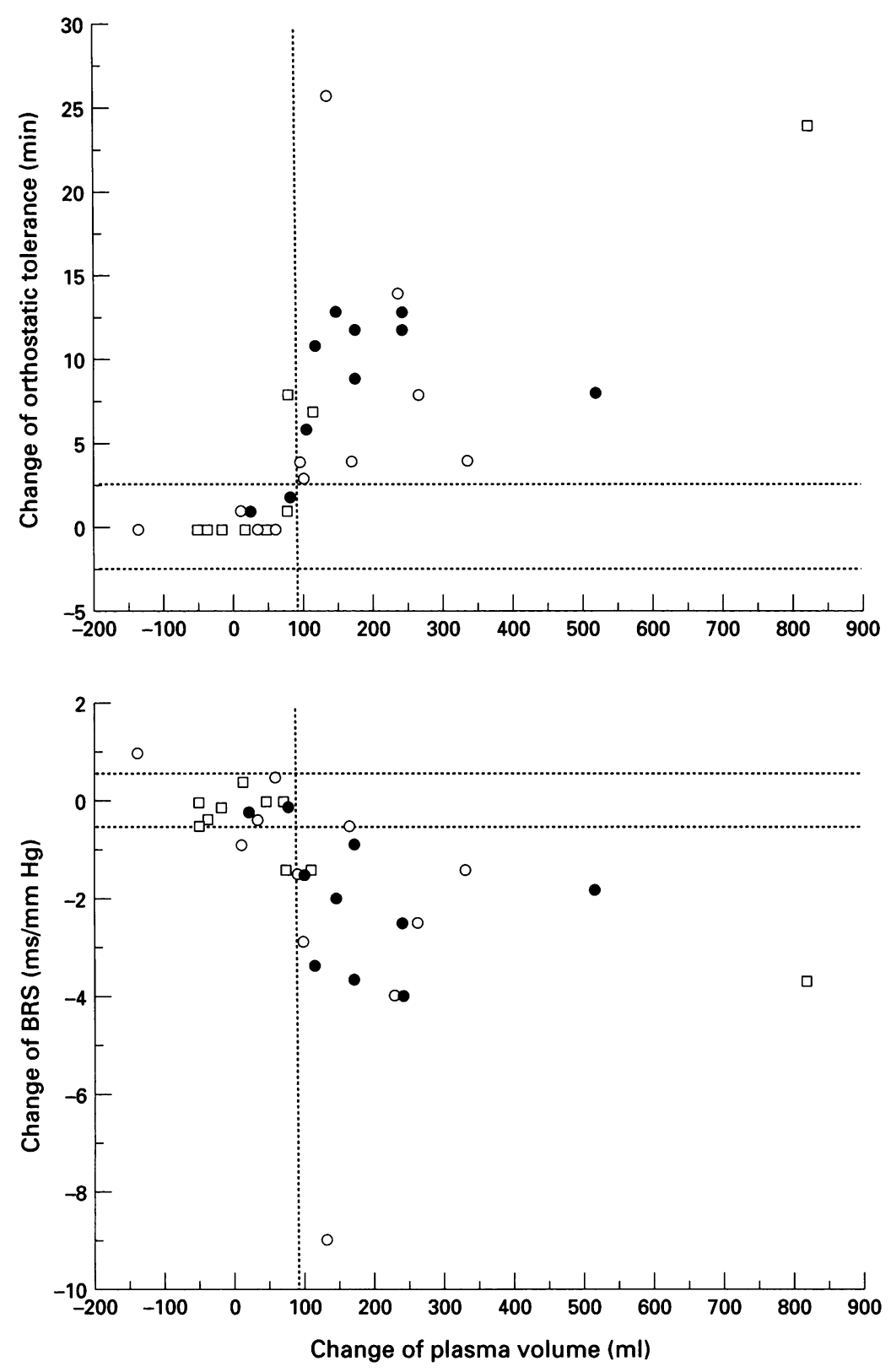

Figure 4 Data from all patients showing the relations between the changes in plasma volume and the changes in orthostatic tolerance (top) and the changes in plasma volume and the changes in baroreceptor sensitivity (BRS) (bottom). Closed circles are from the double blind salt group, open circles from the open salt group, and squares from the placebo group. Note that changes in plasma volume of over $90 \mathrm{ml}$, in all groups of patients, are invariably associated with increases in orthostatic tolerance and decreases in baroreceptor sensitivity
To reduce the likelihood of patients changing their diets spontaneously, we were careful not to mention salt to any of our patients and referred to the treatment only as a "mineral dietary supplement". The lack of dietary control in these patients is clearly a limitation to the study. However, our purpose was not to make a detailed study of sodium balance, but rather to determine whether a large dietary salt supplement on top of the normal intake could increase the plasma and blood volumes and improve orthostatic tolerance. The fact that three patients spontaneously increased salt intake and that this was accompanied by increases in the blood volume and orthostatic tolerance adds further support to our conclusions.

The results showed that six $(30 \%)$ of the patients taking salt did not show any significant responses. The lack of response was related to their initial salt intake as reflected in the 24 hour urinary excretion. Those patients who already took a large quantity of salt in their diets did not show increases in volume to further salt loading. Many of the patients who did show good responses volunteered that they had avoided salt with their meals and often also in cooking. The relation that we observed between salt and plasma volume is actually entirely compatible with the results of earlier salt balance studies ${ }^{172223}$ that showed that low dietary sodium was associated with low extracellular fluid volumes and that after an increase in sodium intake there was some retention of salt and water before a new balance was achieved when the input was excreted. The relation between input and volume change, however, is not linear and at high salt loads little extra volume is retained. ${ }^{1724}$ These observations explain our findings that salt loading had an effect only on relatively salt depleted individuals.

Blood volume was calculated from plasma volume using the haematocrit. There was no significant change in the haematocrit in these patients after eight weeks of salt treatment, which implies that the red cell volume must also have increased. Reticulocytes were counted before and after treatment to test for haemopoiesis and, though some patients showed increases, overall the change was not significant. This may have been because the haemopoiesis was almost complete after eight weeks and also because reticulocytosis is said to occur in bursts ${ }^{25}$ and we may have missed the peaks in many patients.

The striking and consistent findings from this work were the associations between the responses of plasma and blood volumes and the changes in both orthostatic tolerance and baroreceptor sensitivity. These associations were seen in all groups of patients including the three patients taking placebo who showed increases in their volumes. This finding is entirely compatible with our recent report showing a highly significant positive correlation in a large group of patients between orthostatic tolerance and plasma volume and a negative correlation with baroreceptor sensitivity. ${ }^{16}$ also showed increases in urinary sodium excretion at the second assessment and this indicates that all three had increased their salt intakes.

and decreased baroreceptor sensitivity. patients given salt and three out of 10 of placebo in the double blind study and seven Interestingly, the patient who showed the largest change in the entire study was actu one who was given placebo. However, it transpontaneously and had had changed her diet body weight as well as in plasma and blood 

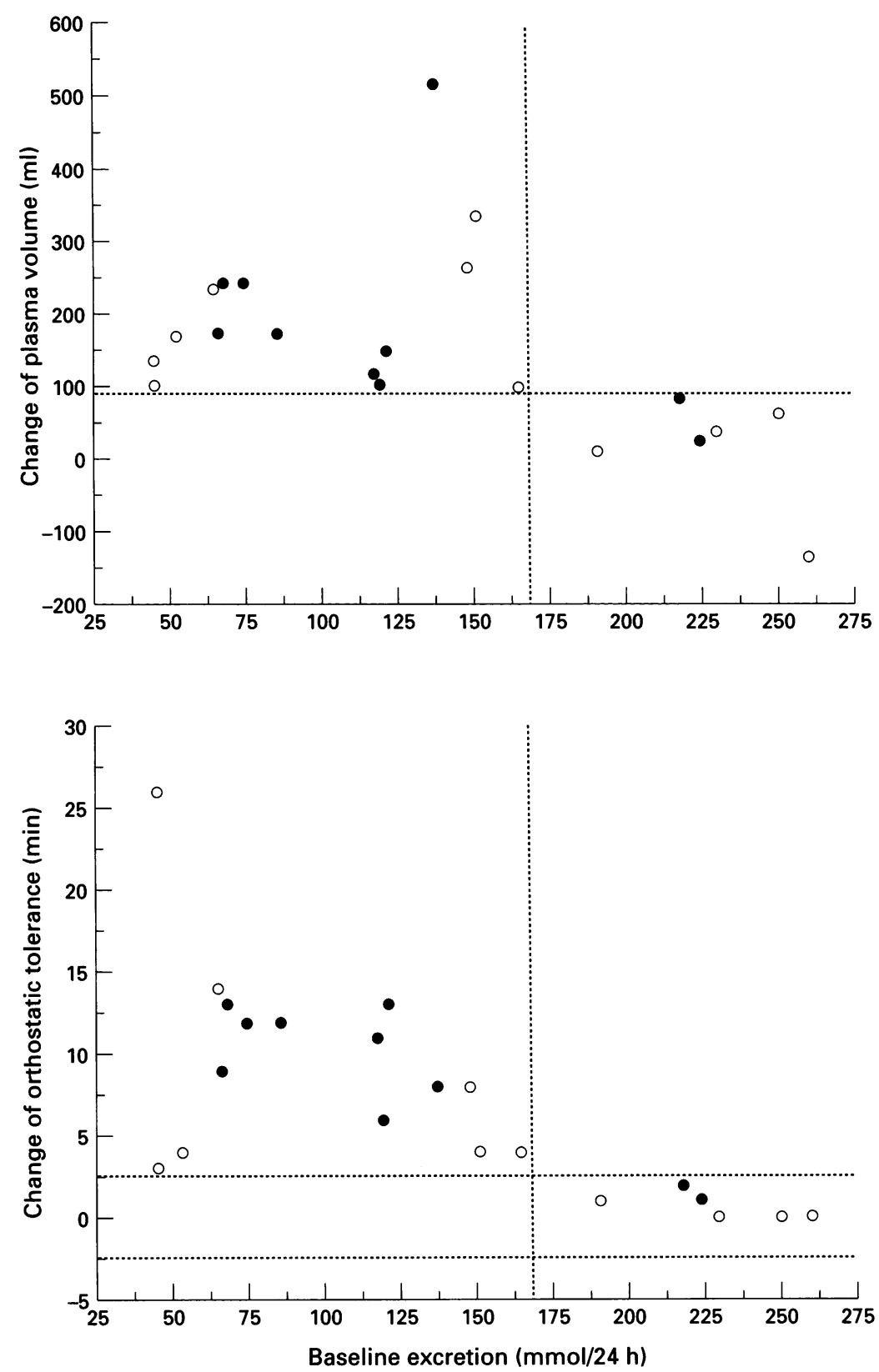

Figure 5 Relation between the pretreatment 24 hour urinary excretion of sodium and the response of plasma volume (top) and orthostatic tolerance (bottom) to salt administration. Closed circles give data from the double blind salt group and open circles from the open study group. Note that only patients in whom the initial salt excretion was less than 170 mmol/24 hours showed significant improvement after salt administration

The effect of plasma volume on orthostatic tolerance is not unexpected. The stress in orthostasis is effectively a decrease in circulatory volume due partly to venous distension and partly to capillary transudation which, even with just upright tilting or standing, can cause a loss of about $15 \%$ of plasma volume in 15 minutes. ${ }^{26}$ It is therefore to be expected that, if it is possible to expand an individual's plasma volume, this should result in an increase in his orthostatic tolerance. This is the first report of changes in plasma volume and orthostatic tolerance in patients complaining of attacks of syncope. However, these results are consistent with previous reports that, in normal subjects following prolonged inactivity there were decreases in plasma volume and this was associated with decreases in orthostatic tolerance. ${ }^{27} 28$
The negative associations between the changes in baroreceptor sensitivity and plasma volume and orthostatic tolerance are harder to understand. It must first be emphasised that, in this study, baroreceptor sensitivity refers only to the prolongation of cardiac interval in response to carotid sinus distension. The important factor in resisting orthostatic hypotension is really the increase in vascular resistance to baroreceptor unloading and this was not actually studied. Nevertheless, these results are quite compatible with our earlier work showing negative correlations in a different group of subjects between baroreceptor sensitivity and both plasma volume and orthostatic tolerance. ${ }^{16}$ Furthermore there have been some studies in animals ${ }^{29}{ }^{30}$ which have shown similar associations. Clearly, much more work is needed to establish the role of the baroreceptor reflex in guarding against orthostatic hypotension.

Although this study has shown that increased salt intake is associated with increases in plasma volume and orthostatic tolerance, this does not prove that the increased orthostatic tolerance is necessarily the result of the expanded plasma volume. Syncope occurs when vasoconstriction abruptly fails, ${ }^{31}$ and it is conceivable that salt loading may alter reflex activity in some way which has the effect of delaying the onset of sympathetic inhibition. Such an effect might be related to the observed changes in baroreflex sensitivity.

In assessing patients, with unexplained syncope, we feel that objective measurements, as made in this study, are more reliable than subjective assessments. Clearly, relief of the patients' symptoms is the primary concern and all of our patients who responded to salt claimed to be improved symptomatically. However, postural syncope is sporadic. In this study the median number of attacks was three over a median period of 12 months. This means that, for subjective changes to be reliable, a larger and longer study would be needed.

We believe that the results of this study have important implications for the management of patients with unexplained syncope. It is important to emphasise again that the patients studied for this report were apparently healthy. They had no evidence of cardiovascular, neurological, or renal disease and, apart from occasional attacks of syncope or near syncope, were indistinguishable from the rest of the population. In managing these patients, we advocate first excluding all known organic causes of syncope. Then the patient's urinary excretion of sodium and his tolerance to orthostatic stress should be determined and, unless the sodium excretion is high $(>170$ $\mathrm{mmol} /$ day), oral salt administration should be given. The advantages of this simple treatment are, firstly, that it is effective in people secreting low levels of salt and secondly, it is safe; we did not observe any adverse reactions. There was even no effect on supine blood pressure although it would be advisable, particularly in elderly subjects, to review patients at intervals 
to ensure that supine blood pressure does not subsequently increase. We do not yet know whether it is necessary to give as much as 120 mmol of salt per day. However, until we know otherwise it is reasonable to start with this dose and possibly to reduce it later to a lower maintenance level.

This work was supported by the British Heart Foundation.

1 Day SC, Cook EF, Funchenstein H, Goldman L. Evaluation and outcome of emergency room patients with transient loss of consciousness. $\mathrm{Am} \mathcal{f} \mathrm{Med}$ 1982;73:15-23.

2 Wahbha MMAE, Morley CA, Al-Shamma YMH, Hainsworth $R$. Cardiovascular reflex responses in
patients with unexplained syncope. Clin Sci 1989;77: 547-53.

3 Waxman MB, Yao L, Cameron DA, Wald RW, Roseman $\mathrm{J}$. Isoproterenol induction of vasodepressor-type reaction in vasodepressor-prone persons. Am f Cardiol 1989, 63:58-65.

4 Fitzpatrick AP, Theodorakis G, Vardas P, Sutton R Methodology of head-up tilt testing in patients with

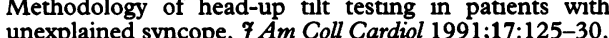

5 El-Bedawi KM, Hainsworth R. Combined head-up tilt and lower body suction: a test of orthostatic tolerance. Clin Auton Res 1994;4:41-7.

6 Hainsworth R, El-Bedawi KM. Orthostatic tolerance in patients with unexplained syncope. Clin Auton Res 1994;4:239-44.

7 El-Bedawi KM, Hainsworth R. Cardiac pacing does no improve patients with vasovagal syncope. Clin Auton Res 1994;4:233-37.

8 Goldenberg IF, Almquist A, Dunbar DN, Milstein S, Pritzker MR, Benditt DG. Prevention of neurally-mediated syncope by selective $\beta_{1}$-adrenoreceptor blockade Circulation 1987;76 Suppl IV:133.

9 Grubb BP, Temesy-Armos P, Hahn H, Elliot L. Utility of upright tilt table testing in evaluation and management of

10 Asso A, Milstein S, Dunnigan A, Remole S, Bailin S, Benditt DG. Prognostic significance of parenteral metoprolol during head-up tilt-table testing: follow-up after 3 prolol during head-up tilt-table testing: foll

11 Chan WL, Kong CW, Chang MS, Chiang BN. Exacerbation of vasodepressor syncope by beta-adrenergic blockade (letter). N Engl ₹ Med 1991;324:1219-20.

12 Frietas J, Puig J, Campos J, Garcia JM, Cunha DI Carvalho MJ, et al. Malignant vasovagal syncope: a case of prolonged asystole induced by "tilt" test and aggravated by therapy with beta-blockers (a clinical case and diagnostic, physiopathologic and therapeutic review) Rev Portug Cardiol 1993;12:745-51.

13 Rashtian MY, Bhandari AK. Worsening of neurocardiogenic syncope by beta-blockers. Ann Intern Med 1993; 119:955-6.
14 Sneddon JF, Camm AJ. Vasovagal syncope: classification, investigation and treatment. $\mathrm{Br} \mathcal{F}$ Hosp Med 1993;49: 329-34.

15 Edmonds ME, Watkins PJ Clinical presentation of diabetic autonomic failure. In: Bannister R, Mathias CJ, eds. Autonomic failure. A textbook of clinical disorders of the autonomic nervous system. Oxford: Oxford University autonomic nervous syst

16 El-Sayed H, Hainsworth R. Relationship between plasma volume, carotid baroreceptor sensitivity and orthostatic tolerance. Clin $S c i$ 1995;88:463-70.

17 Gupta BN, Linden RJ, Mary DASG, Weatherill D. The influence of high and low sodium intake on blood volume in dogs. Q F Exp Physiol 1981;66:117-28.

18 Brown WJ, Brown FK, Krishan I. Exchangeable sodium and blood volume in normotensive and hypertensive humans on high and low sodium intake. Circulation 1971;43:508-19.

19 El-Sayed H, Goodall S, Hainsworth R. Reevaluation of Evans blue dye dilution method of measurement of plasma volume. Clin Lab Haematol 1995;17:189-94.

20 International Committee for Standardization in Haematology. Standard techniques for the measurement of red cell and plasma volume: panel on diagnostic application of radioisotopes in haematology. Br F Haematol 1973;25: 801-14.

21 Al-Timman JKA, Hainsworth R, Vukasovic JL. A modified neck chamber for the study of carotid baroreceptors in neck chamber for the study of
humans. $¥$ Physiol $1988 ; 399: 14 P$.

22 Lyons RH, Jacobson SD, Avery NL. Increases in the plasma volume following the administration of sodium plasma volume following the admini
salts. $A m \mathcal{F} M e d S c i$ 1944;208:148-54.

23 Romero JC, Staneloni RJ, Dufau JL, Dohmen R, Binia A, Kliman B, et al. Changes in fluid compartments, renal induced by low sodium intake. Metabolism 1968;17:10-9. 24 O'Connor WJ. Normal renal function. London: Croom Helm, 1982;299-328.

25 Lange RD, Jones JB. Erythropoiesis in dogs and humans with cyclic haemotapoiesis. In: Dunn CDR, ed. Current concepts in erythropoiesis. Chichester: John Wiley 143-65.

26 Hagan RD, Diaz FJ, Horvath SM. Plasma volume changes with movement to supine and standing position. $7 \mathrm{Appl}$ Physiol 1978;45:414-8.

27 Bungo MW, Charles JB, Johnson PC. Cardiovascular deconditioning during spaceflight and the use of saline as a counter measure to orthostatic intolerance. Aviat Space Environ Med 1985;56:985-90.

28 Ludwig D, Convertino VA. Predicting orthostatic intolerance: physics or physiology? Aviat Space Environ Med 1994;65:404-11.

29 Vatner SF, Boettcher DH, Hayndrickx GR, McRitchie RJ. Reduced baroreflex sensitivity with volume loading in conscious dogs. Circ Res 1975;37:236-42.

30 Billman GE, Dickey DT, Teho KK, Stone HL. Effects of central venous blood volume shifts on arterial baroreflex control of heart rate. Am $\mathcal{F}$ Physiol 1982;241:H571-5.

31 Barcroft $\mathrm{H}$, Edholm OG. On the vasodilatation in human skeletal muscle during post-haemorrhagic fainting. $f$ Physiol 1945;104:161-75. 